\title{
Psychological characteristics of religious delusions
}

\author{
Robel Iyassu • Suzanne Jolley • Paul Bebbington • Graham Dunn • \\ Richard Emsley • Daniel Freeman • David Fowler • Amy Hardy • \\ Helen Waller · Elizabeth Kuipers · Philippa Garety
}

Received: 30 May 2013/Accepted: 16 December 2013/Published online: 31 December 2013

(c) Springer-Verlag Berlin Heidelberg 2013

\begin{abstract}
Purpose Religious delusions are common and are considered to be particularly difficult to treat. In this study we investigated what psychological processes may underlie the reported treatment resistance. In particular, we focused on the perceptual, cognitive, affective and behavioural mechanisms held to maintain delusions in cognitive models of psychosis, as these form the key treatment targets in cognitive behavioural therapy. We compared religious delusions to delusions with other content.

Methods Comprehensive measures of symptoms and psychological processes were completed by 383 adult participants with delusions and a schizophrenia spectrum diagnosis, drawn from two large studies of cognitive behavioural therapy for psychosis.

Results Binary logistic regression showed that religious delusions were associated with higher levels of grandiosity
\end{abstract}

R. Iyassu · S. Jolley $(\bowtie) \cdot$ A. Hardy $\cdot$ H. Waller $\cdot$ E. Kuipers . P. Garety

PO77 Department of Psychology, King's College London, Institute of Psychiatry, University of London, Denmark Hill, London SE5 8AF, UK

e-mail: suzanne.jolley@kcl.ac.uk

P. Bebbington

Department of Mental Health Sciences, UCL, London, UK

G. Dunn · R. Emsley

Health Sciences Research Group, School of Community Based

Medicine, University of Manchester, Manchester, UK

D. Freeman

Department of Psychiatry, University of Oxford, Oxford, UK

D. Fowler

School of Medicine, Health Policy and Practice,

University of East Anglia, Norwich, UK
(OR 7.5; $95 \%$ CI 3.9-14.1), passivity experiences, having internal evidence for their delusion (anomalous experiences or mood states), and being willing to consider alternatives to their delusion (95\% CI for ORs 1.1-8.6). Levels of negative symptoms were lower. No differences were found in delusional conviction, insight or attitudes towards treatment.

Conclusions Levels of positive symptoms, particularly anomalous experiences and grandiosity, were high, and may contribute to symptom persistence. However, contrary to previous reports, we found no evidence that people with religious delusions would be less likely to engage in any form of help. Higher levels of flexibility may make them particularly amenable to cognitive behavioural approaches, but particular care should be taken to preserve self-esteem and valued aspects of beliefs and experiences.

Keywords Psychosis - Schizophrenia $\cdot$ CBT $\cdot$ Cognitive model

\section{Introduction}

Delusions are a cardinal feature of psychotic illness, present in around three quarters of people with a schizophrenia spectrum diagnosis [1, 2]. Religious themes are common across delusion categories and types, with between a fifth and two-thirds of all delusions reflecting religious content [3-6]. To be classified as a religious delusion, the belief must be idiosyncratic, rather than accepted within a particular culture or subculture [7]. Strongly held beliefs that are shared within an existing religious or spiritual context would not, therefore, be considered to be religious delusions, irrespective of cooccurring psychosis. For example, believing oneself to be 
able to hear the voice of Jesus is not uncommon in a Christian society and thus would not in itself be classified as a religious delusion. In contrast, believing oneself to be inhabited by the warring spirits of multiple interspatial deities, would be considered to be a religious delusion. Culturally acceptable religious beliefs are cited as an important coping strategy for many people with schizophrenia, and may contribute to lower symptom severity in both severe and enduring mental illnesses [8, 9] and common mental disorders [10, 11]. Religious delusions, in contrast, have routinely been linked to poorer prognosis for people with psychotic disorders [12].

Levels of disability, distress and conviction have all been reported to be higher in people with religious delusions compared to other types of delusions [1, 3, 4, 13-15]. Religious delusions are also associated with poor engagement, low satisfaction with services and with treatment, and longer duration of untreated psychosis [12, 16-19]. People with religious delusions appear, therefore, to be a particularly problematic group to treat effectively, and ought to be targeted for psychological therapies [20, 21]. However, as the mechanisms underlying the treatment resistance are poorly understood, further study is required to establish what the particular foci of psychological intervention for people with religious delusions should be, and what issues are likely to arise in implementation.

Cognitive models of psychosis [22, 23] identify specific psychological maintaining factors for delusions. Prominent amongst these are persisting anomalous experiences, reasoning biases, affective processes, and poor adjustment to psychosis resulting from personal beliefs about illness, treatment and recovery. Religious delusions can be plausibly linked to increased difficulty in all these areas.

Anomalous experiences These may be perceived as having religious significance (e.g., communications from higher powers) and thus be specifically attended to, engaged with and even deliberately induced. Frequent anomalous experiences provide repeated evidence to sustain the delusion.

Reasoning biases Delusions are considered to arise from, and be maintained by, biases and errors in evidencebased reasoning. These include 'jumping to conclusions' (JTC) by making decisions based on limited data, and belief inflexibility, comprising difficulty adjusting beliefs in response to contradictory evidence; difficulty considering the possibility of being mistaken; and difficulty identifying plausible alternative explanations [24]. Faith, by its nature, relies on foundations other than a systematic and evolving evidence base, and religious or spiritual insights tend to be based on revelation, dramatic events or inner conviction, rather than a process of hypothesis testing. It is also common, and, in some religions, even desirable, for religious beliefs to be held with high conviction, certainty of rectitude (rather than possibility of being mistaken), and without alternatives. Should these features of religious beliefs equally characterise delusions with religious content, reasoning biases may be particularly prominent, and thus contribute to severity, persistence, and higher levels of conviction.

Affective disturbance Affective processes are implicated in the onset and maintenance of delusions by their impact on attentional, perceptual, interpretative and memory processes, and through maladaptive coping and affect regulation strategies [25]. Religious delusions, by definition, concern themes of universal existential import, and are therefore likely to be particularly associated with strong affect, with consequent cognitive-perceptual and behavioural changes which may act to further increase delusion severity [26].

Beliefs about illness, treatment and recovery How a person makes sense of the changes associated with psychosis is important to their adjustment and to their engagement with treatment [27, 28]. Religious delusions may be particularly likely to involve a rationale at odds with the tradition of Western psychiatric empiricism that characterises mental health services in the UK. This mismatch of explanatory models may underpin the association of religious delusions with poor engagement with treatment and with services [28, 29].

\section{Aims of the current study}

We set out to compare a large sample of people with religious delusions to people with other kinds of delusions to identify the psychological factors which may contribute to the increased persistence, disability and distress reported to be associated with religious delusions. All participants had current delusional symptomatology, and a schizophrenia spectrum diagnosis verified by trained assessors. The aim was to develop a better psychological understanding of religious delusions to inform model development and, thereby, intervention.

We tested the following specific hypotheses:

1. In line with previous studies, people with religious delusions will have higher levels of symptomatology and delusional conviction, and poorer engagement in treatment than people with other kinds of delusions.

2. People with religious delusions will have more anomalous experiences, more negative affect and more reasoning biases than people with other kinds of delusions.

3. People with religious delusions will have less insight and more unhelpful attitudes towards their treatment than people with other kinds of delusions. 


\section{Methods}

Participants

Participants in the present study were the combined samples from two studies by the psychosis research partnership. The first was the psychological prevention of relapse in psychosis trial (PRP, ISRCTN83557988). The PRP trial was a United Kingdom multicentre randomised controlled trial of cognitive behavioural therapy and family intervention for psychosis [30]. The second was the cognitive mechanisms of change in delusions (CMCD, ISRCTN 59501939) study. Both studies had ethical approval (South East REC ref. 01/1/14; London Wandsworth REC ref. 07/H0803/140). Participants were recruited from National Health Service Trusts in London and East Anglia and gave informed consent prior to participation. The two studies used the same inclusion criteria: a current diagnosis of nonaffective psychosis according to ICD-10 criteria (F20-29) as assessed by trained raters using the schedules for clinical assessment in neuropsychiatry (SCAN) [31]; aged between 18 and 65 years; positive psychotic symptoms of at least moderate severity at the point of recruitment (as rated by the SCAN). For the PRP trial, participants had to have experienced at least one relapse; for the CMCD study, the positive symptom needed to be a distressing delusion, held with at least $50 \%$ conviction over the last 3 months. The following exclusions were applied in both studies: primary diagnosis of alcohol or substance dependency; organic syndrome or learning disability; inadequate command of English to engage in assessments or psychological therapy with an English speaking therapist; and, finally, unstable residential arrangements (possibility of moving away before the study end). A total of 424 participants were included; 301 from the PRP trial, and the first baseline cohort of 130 from the CMCD study (seven participants took part in both studies, and they were excluded from the PRP dataset, so that only their most recent data from the CMCD trial were included in the current study). Of these, 383 had a current delusion (global delusion rating $>1$ on the SAPS, see below) and formed the sample for the current study.

Measures

\section{Symptom severity}

The scales for the assessment of positive and negative symptoms $[32,33]$ were used to measure symptom severity over the previous month in the following domains: hallucinations, delusions, bizarre behaviour, positive formal thought disorder, affective flattening, alogia, avolitionapathy, anhedonia-asociality, and attention. Each domain includes a global rating of severity, rated by an interviewer on a Likert scale in the following way: $0=$ none, 1 = questionable, $2=$ mild, $3=$ moderate, $4=$ marked and $5=$ severe. For this study, the global ratings of each domain were summed to give an indication of symptom severity. Both of the measures are widely used, and have good psychometric properties. The global rating of delusions (item 20) was used to select participants with any kind of delusion from the total sample, and the religious delusions item (item 12) to identify whether or not participants had a religious delusion. In each case, a rating of 2 (mild severity) or more was taken to indicate presence of the delusion. Delusional conviction was rated using the anchoring from the Psychotic Symptoms Rating Scales (PSYRATS, [34]): $0=$ no conviction at all, $1=$ very little conviction $(<10 \%), 2=$ some doubts in conviction$(10-49 \%), 3=$ conviction belief is very strong, between 50 and $99 \%$, and $4=100 \%$ conviction. The PSYRATS has good inter-rater reliability (intraclass correlation coefficients range from 0.79 to 1.0 ).

\section{Treatment engagement}

Attitudes towards medication were assessed using the first four items of the Medication Adherence Rating Scale (MARS, [35]). A higher MARS score indicates higher levels of medication adherence. Ratings of engagement in CBT $(2=$ full therapy; $1=$ partial therapy; $0=$ no therapy, [36]) and with services (rated on an 11 item scale, from 1 (poor engagement) to 5 (good engagement), [37]) were available from those patients randomised to the CBT intervention arm of the PRP trial only.

\section{Anomalous experiences}

SCAN ratings were used to create a dichotomous variable denoting whether or not the participant had any hallucinatory experiences in any modality. Ratings were also made of whether the main source of evidence for the delusional belief was internal (a mood state or anomalous experience) or external (an external event).

\section{Affect}

The beck depression inventory-II (BDI-II, [38]) and beck anxiety inventory (BAI, [39]) were used to assess concurrent emotional upset. Both are self-report 21-item, 4-point scales (0-3). Anxiety is assessed over the past week with the following severity ratings: $0-9=$ normal, $10-18=$ mild to moderate, $19-29=$ moderate to severe and $30-63=$ severe. Depression is assessed over the past 2 weeks with the following anchors: 0-13: normal; 14-19: mild depression; 20-28: moderate depression; and 29-63: 
severe depression. Both scales are widely used and have excellent psychometric properties.

\section{Reasoning}

Two aspects of reasoning were assessed, belief flexibility (BF) and the jumping to conclusions data-gathering bias (JTC). BF comprises three components [40]. Two items are from the Maudsley assessment of delusions (MADS, [41]): whether the respondent believes there is a possibility that they may be mistaken in their delusional belief (PM), rated yes or no; and the respondent's reaction to a 'hypothetical contradiction', a convincing scenario which would refute the delusional belief (RTHC), rated flexible (dismisses the delusion, or believes it less) or inflexible (dismisses the evidence, or changes the delusional belief to accommodate the evidence). An additional measure of alternative explanations of experiences (EoE, [42]) assesses whether or not respondents can think of any other explanation at all (except the delusional explanation) for the experiential evidence they have listed in support of their delusion. The MADS is a validated, structured interview schedule designed to assess multiple dimensions of delusions, with good inter-rater and adequate test-retest reliability [43].

JTC was assessed using two versions of the probabilistic reasoning 'beads' task [44], which consists of presentations of beads in a jar (arranged in an 85:15 or 60:40 ratio, using two different colours). Beads are shown one at a time in a predetermined order and participants are instructed to take as many draws as they need to be certain of the jar of origin. The JTC bias is defined dichotomously as a decision after fewer than three beads [40, 44].

\section{Insight and attitudes to treatment}

Personal illness beliefs were assessed using two subscales of the Illness Perceptions Questionnaire (IPQ, [45]): timeline (three items) and possibility of cure-control (six items), together with the two 'internal' cause items, relating to 'state of mind' and 'personality'. These items have been demonstrated to predict the uptake of psychological therapy [28]. Each item is rated from 1 (strongly disagree) to 5 (strongly agree), and psychometric properties are good. Insight was measured using the first three items of the scale to assess unawareness of mental disorder [46]. Each item was rated from 1 (good insight) to 5 (poor insight). The scale is interviewer rated with good psychometric properties.

\section{Analysis}

Analyses were conducted using IBM SPSS 20 for windows (version 20). Significance tests were two-tailed. Item 12 of the SAPS (religious delusions, RD) was used to dichotomise the sample for the main analyses, using a cut off of 2 (ratings of 'mild' and above) to indicate the presence of a religious delusion (RD group) or the absence of religious delusions, but presence of any other delusion (other delusions group). The association between delusions of other types and religious delusions was assessed by binary logistic regression, with the categorical coding of religious delusions as the dependent variable and each remaining delusion type as predictors. To assess differences between participants with and without religious delusions, a series of independent samples $t$ tests were computed. Where two dichotomous variables were tested against each other, Chi Square $\left(\chi^{2}\right)$ tests were computed. Parametric assumptions were met for all analyses with the exception of the $t$ test for the SANS Alogia score, for which a Satterthwaite adjustment was carried out. Given the exploratory nature of the study, no formal adjustment was made for multiple testing. Significantly different variables were entered into a binary logistic regression, with $\mathrm{RD}$ group $(1=\mathrm{RD}$ group; $0=$ other delusions group) as the dependent variable, using a backward conditional selection procedure, to identify a final model of the correlates of religious delusions.

\section{Results}

Demographic characteristics and prevalence of religious delusions

87 individuals (20.5\%) had religious delusions (RD). The RD group did not differ from the other delusions group on any demographic variable (Table 1). The prevalence rates of all types of delusions in the sample are displayed in Table 2. Binary logistic regression analyses revealed that, compared to the other delusions group, and irrespective of controlling for all other delusion types, people with $\mathrm{RD}$ were six times more likely to also have grandiose delusions, and three times more likely to also experience delusions of being controlled (Table 2).

Hypothesis 1 People with religious delusions will have more severe symptoms, higher delusional conviction and show poorer engagement with treatment than people with other delusions.

The RD group was characterised by higher levels of positive symptoms, but lower levels of negative symptoms, and similar levels of conviction, compared to those with other delusions. The RD group scored more highly on hallucinations, bizarre behaviour, formal thought disorder, and, in negative symptoms, lower on alogia, avolition/ apathy and anhedonia/asociality. Effect sizes were small to 
Table 1 Demographic and clinical characteristics of participants with religious delusions compared to those with other delusions

\begin{tabular}{|c|c|c|c|c|c|c|c|c|c|}
\hline & \multicolumn{2}{|c|}{ Total sample $(n=383)$} & \multicolumn{2}{|c|}{ Religious delusions $(n=87)$} & \multicolumn{2}{|c|}{ Other delusions $(n=296)$} & \multicolumn{2}{|c|}{$t(d f)$} & $p$ \\
\hline \multicolumn{10}{|l|}{ Age (years) } \\
\hline Mean (SD) & \multicolumn{2}{|l|}{$38.9(11.3)$} & \multicolumn{2}{|l|}{$38.9(10.6)$} & \multicolumn{2}{|c|}{$38.9(11.7)$} & \multicolumn{2}{|c|}{$-0.03(381)$} & 1.0 \\
\hline \multicolumn{10}{|c|}{ Length of illness (years) } \\
\hline \multirow[t]{2}{*}{ Mean (SD) } & \multirow[t]{2}{*}{$11.9(9.6)$} & & \multicolumn{2}{|l|}{$13.3(10.4)$} & \multicolumn{2}{|c|}{$11.5(9.4)$} & \multicolumn{2}{|c|}{$-1.5(375)$} & 0.1 \\
\hline & & Total sam & $(n=383)$ & Religious delusio & $\imath=87)$ & Other delusions ( & 296) & $\chi^{2}(d f)$ & $p$ \\
\hline \multicolumn{10}{|l|}{ Sex, $n(\%)$} \\
\hline \multicolumn{2}{|l|}{ Male } & $266(69)$ & & $60(69)$ & & $206(70)$ & \multirow{2}{*}{\multicolumn{2}{|c|}{$0.01(1)$}} & 0.9 \\
\hline Female & & $117(31)$ & & $27(31)$ & & $90(30)$ & & & \\
\hline \multicolumn{10}{|c|}{ Ethnicity, $n(\%)$} \\
\hline \multicolumn{2}{|l|}{ White } & $264(69)$ & & $63(72)$ & & $201(68)$ & \multirow{3}{*}{\multicolumn{2}{|c|}{$0.7(2)$}} & 0.7 \\
\hline Black Africa & aribbean/other & $76(20)$ & & $16(18)$ & & $60(20)$ & & & \\
\hline Asian/other & & $43(11)$ & & $8(10)$ & & $35(12)$ & & & \\
\hline \multicolumn{10}{|c|}{ Diagnosis, $n(\%)$} \\
\hline \multicolumn{2}{|c|}{ Schizophrenia } & $327(85)$ & & $69(80)$ & & $258(87)$ & \multirow{3}{*}{\multicolumn{2}{|c|}{$4.3(2)$}} & 0.2 \\
\hline Schizoaffecti & lisorder & $43(11)$ & & $15(17)$ & & $28(9)$ & & & \\
\hline Delusional d & ler/other & $11(4)$ & & $2(2)$ & & $9(4)$ & & & \\
\hline \multicolumn{10}{|c|}{ Medication, $n(\%)^{\mathrm{a}}$} \\
\hline \multicolumn{2}{|l|}{ None } & $8(2)$ & & $1(1)$ & & $7(2)$ & \multirow{3}{*}{\multicolumn{2}{|c|}{$5.0(3)$}} & 0.2 \\
\hline Low & & $119(32)$ & & $27(32)$ & & $92(32)$ & & & \\
\hline Medium & & $134(36)$ & & $24(29)$ & & $110(38)$ & & & \\
\hline High & & $109(29)$ & & $32(38)$ & & 77 (27) & & & \\
\hline
\end{tabular}

${ }^{a}$ Chlorpromazine equivalent, $0-200=$ low; $201-400=$ medium; $>400=$ high

Table 2 Binary logistic regression showing the prevalence of delusional subtypes between participants with religious delusions compared to those with other delusions (with percentage prevalence rates for each group)

$O R$ odds ratio, $C I$ confidence intervals

${ }^{a}$ Uncontrolled analyses suggest

$\mathrm{RD}$ group less likely to

experience persecutory

delusions (OR 0.4, $95 \% \mathrm{CI}$

$0.3-0.8, p=0.004)$

b No association in uncontrolled analyses (OR 1.5, $95 \%$ CI $0.7-2.9, p=0.2$ )

\begin{tabular}{llcllll}
\hline Delusion subtype & \multicolumn{2}{l}{ Age prevalence $(\%)$} & OR & $95 \%$ CI & $p$ \\
\cline { 2 - 6 } & $\begin{array}{l}\text { Total sample } \\
(n=383)\end{array}$ & $\begin{array}{c}\text { Religious } \\
\text { delusions } \\
(n=87)\end{array}$ & $\begin{array}{c}\text { Other delusions } \\
(n=296)\end{array}$ & & & \\
& 80 & 69 & 83 & $0.7^{\mathrm{a}}$ & $0.3-1.2$ & 0.2 \\
\hline Persecutory delusions & 68 & 71 & 67 & 1.0 & $0.5-1.8$ & 0.9 \\
Delusions of reference & 40 & 48 & 38 & 1.4 & $0.8-2.5$ & 0.3 \\
Delusions of mind reading & 12 & 16 & 11 & $2.2^{\mathrm{b}}$ & $1.0-4.8$ & 0.04 \\
Delusions of sin and guilt & 30 & 59 & 21 & 6.2 & $3.5-11.1$ & $<0.001$ \\
Grandiose delusions & 21 & 100 & 0 & & & \\
Religious delusions & 20 & 26 & 19 & 1.1 & $0.5-2.4$ & 0.8 \\
Thought insertion & 19 & 21 & 18 & 1.0 & $0.5-2.1$ & 0.8 \\
Somatic delusions & 18 & 21 & 17 & 0.9 & $0.4-2.0$ & 0.8 \\
Thought broadcast & 18 & 29 & 15 & 3.1 & $1.5-6.2$ & 0.002 \\
Delusions of being controlled & 7 & 10 & 6 & 1.2 & $0.4-3.3$ & 0.8 \\
Thought withdrawal & 2 & 1 & 2 & 0.6 & $0.1-5.8$ & 0.7 \\
Delusions of jealousy & & & & &
\end{tabular}

medium. The RD group was as likely to engage with services, with talking therapy, and with medication as those with other delusions (Table 3).
Hypothesis 2 People with religious delusions will have more anomalous experiences, more negative affect and more reasoning biases than people with other delusions. 
Table 3 Psychotic symptoms, delusional conviction and engagement scores in participants with religious delusions compared to those with other types of delusions
SAPS/SANS scale for the assessment of positive/negative symptoms, PSYRATS Psychotic Symptoms Rating Scales, MARS Medication Adherence Rating Scale, $C B T$ cognitive behavioural therapy, ES effect size, Cohen's $d$ [55]

${ }^{\text {a }}$ Only available for data from CBT intervention arm of PRP trial

b Satterthwaite adjustment carried out

\begin{tabular}{|c|c|c|c|c|c|c|}
\hline \multicolumn{2}{|l|}{ Variable } & $\begin{array}{l}\text { Religious } \\
\text { delusions } \\
(n=87) \\
\text { Mean (SD) }\end{array}$ & $\begin{array}{l}\begin{array}{l}\text { Other } \\
\text { delusions } \\
(n=296) \\
\text { Mean (SD) }\end{array}\end{array}$ & $t, \mathrm{ES}(d)$ & $d f$ & $p$ \\
\hline \multicolumn{7}{|c|}{ SAPS positive symptoms } \\
\hline \multicolumn{2}{|c|}{ SAPS total } & $9.3(2.7)$ & $8.0(3.1)$ & $-3.5,0.4$ & 378 & $<0.001$ \\
\hline \multicolumn{2}{|c|}{ Hallucinations } & $3.1(1.7)$ & $2.6(1.8)$ & $-2.2,0.3$ & 379 & 0.03 \\
\hline \multicolumn{2}{|c|}{ Delusions } & $4(0.8)$ & $3.8(0.8)$ & $-1.9,0.2$ & 381 & 0.06 \\
\hline \multicolumn{2}{|c|}{ Bizarre behaviour } & $1.1(1.1)$ & $0.7(1.0)$ & $-2.6,0.4$ & 381 & $<0.01$ \\
\hline \multicolumn{2}{|c|}{ Formal thought disorder } & $1.2(1.2)$ & $0.9(1.3)$ & $-2.0,0.3$ & 380 & $<0.05$ \\
\hline \multicolumn{7}{|c|}{ SANS negative symptoms } \\
\hline \multicolumn{2}{|c|}{ SANS total } & $6.7(4.3)$ & $7.9(4.4)$ & $2.3,-0.3$ & 379 & 0.02 \\
\hline \multicolumn{2}{|c|}{ Affective flattening } & $1.0(1.4)$ & $1.1(1.3)$ & 0.4 & 381 & 0.7 \\
\hline Alogia & & $0.4(0.9)$ & $0.6(1.1)$ & $1.8,-0.2$ & $168^{\mathrm{b}}$ & 0.04 \\
\hline Anhedo & & $2.1(1.5)$ & $2.4(1.5)$ & $2.0,-0.2$ & 380 & 0.04 \\
\hline Attentio & & $1.2(1.1)$ & $1.4(1.6)$ & 1.3 & 380 & 0.2 \\
\hline Avolitic & & $2.0(1.4)$ & $2.4(1.4)$ & $2.2,-0.3$ & 381 & 0.03 \\
\hline Convictio & & & & & & \\
\hline PSYRA & & $3.3(0.9)$ & $3.1(1.1)$ & -1.5 & 376 & 0.1 \\
\hline & & $\begin{array}{l}\text { Religious } \\
\text { delusions } \\
(n=87) \\
\text { Mean (SD) } \\
(n=49)\end{array}$ & $\begin{array}{l}\text { Other } \\
\text { delusions } \\
(n=296) \\
\text { Mean (SD) } \\
(n=137)\end{array}$ & $t, \mathrm{ES}(d)$ & $d f$ & $p$ \\
\hline Engagem & & & & & & \\
\hline Engage & total score ${ }^{\mathrm{a}}$ & $41.2(6.2)$ & $40.9(6)$ & -0.3 & 184 & 0.89 \\
\hline MARS & & $2.8(1.3)$ & $2.9(1.2)$ & 1.1 & 353 & 0.3 \\
\hline Variable & $\begin{array}{l}\text { Religious } \\
\text { delusions } \\
(n=87) \\
\text { Mean (SD) } \\
(n=21)\end{array}$ & $\begin{array}{l}\text { Oth } \\
\text { delt } \\
(n= \\
\mathrm{Me} \\
(n=\end{array}$ & $\begin{array}{l}\text { er } \\
\text { sions } \\
=296) \\
\text { an (SD) } \\
=66)\end{array}$ & $\chi^{2}$ & $d f$ & $p$ \\
\hline Uptake o & $(\%)$ & & & & & \\
\hline None & $6(28.6)$ & 14 & (21.2) & 0.6 & 2 & 0.7 \\
\hline Some & 7 (33.3) & 27 & 40.9) & & & \\
\hline Full & $8(38.1)$ & 25 & (37.9) & & & \\
\hline
\end{tabular}

The RD group was more likely to have hallucinations in any modality, and to have internal, rather than external evidence for their delusions. Effect sizes were small. Levels of negative affect were similar between groups. The RD group did not show more severe reasoning biases than those with other delusions, rather, they were slightly more likely to have access to an alternative explanation (Table 4).

Hypothesis 3 People with religious delusions will be characterised by less insight and more negative attitudes towards treatment than people with other delusions.

The RD group did not differ from the Other Delusions group on any insight or illness perception subscore, or on the total scores (Table 4). Scores on these attitudinal measures of engagement were consistent, therefore, with the results for the actual take-up of treatment, as tested in hypothesis One.

Post hoc analysis: correlates of religious delusions

The categorical variables of grandiose delusions, delusions of being controlled, access to an alternative explanation, and having internal evidence for the delusion were entered into a Binary logistic regression analysis, together with the global ratings of hallucinations, bizarre behaviour, formal thought disorder, alogia, anhedonia/asociality, and avolition/apathy, with religious/other delusion as the dependent 
Table 4 Anomalous experiences, affect and reasoning biases in religious delusions compared to other types of delusions
$B D I$ beck depression inventory, $B A I$ beck anxiety inventory, JTC jumping to conclusions, $I P Q$ Illness Perceptions Questionnaire, ES effect size, $r$ [55]

a Only available for data from PRP trial

\begin{tabular}{|c|c|c|c|c|c|c|}
\hline Variable & \multicolumn{2}{|c|}{$\begin{array}{l}\text { Religious delusions }(n=87) \\
n(\%)\end{array}$} & $\begin{array}{l}\text { Other delusions }(n=296) \\
n(\%)\end{array}$ & $\chi^{2}, \mathrm{ES}(r)$ & $d f$ & $p$ \\
\hline \multicolumn{7}{|c|}{ Anomalous experiences } \\
\hline Yes & \multicolumn{2}{|c|}{$66(75.9)$} & $182(61.7)$ & $5.9,0.1$ & 1 & 0.02 \\
\hline No & \multicolumn{2}{|c|}{$21(24.1)$} & $113(38.3)$ & & & \\
\hline \multicolumn{7}{|l|}{ Internal state } \\
\hline Yes & \multicolumn{2}{|c|}{$72(90)$} & 204(80.3) & $4.0,0.1$ & 1 & 0.05 \\
\hline No & \multicolumn{2}{|c|}{$8(10)$} & $50(19.7)$ & & & \\
\hline \multicolumn{7}{|l|}{ External state } \\
\hline Yes & \multicolumn{2}{|c|}{$46(57.5)$} & $173(68.7)$ & 3.4 & 1 & 0.07 \\
\hline No & \multicolumn{2}{|c|}{$34(42.5)$} & \multicolumn{2}{|l|}{$79(31.3)$} & & \\
\hline Variable & \multicolumn{2}{|r|}{$\begin{array}{l}\text { Religious delusions }(n=87) \\
\text { Mean (SD) }\end{array}$} & $\begin{array}{l}\text { Other delusions }(n=296) \\
\text { Mean }(\mathrm{SD})\end{array}$ & 6) & $d f$ & $p$ \\
\hline \multicolumn{7}{|l|}{ Affect } \\
\hline \multirow{2}{*}{\multicolumn{2}{|c|}{$\begin{array}{l}\text { Depression (BDI) } \\
\text { Anxiety (BAI) }\end{array}$}} & $21.9(12.4)$ & $23.7(13.4)$ & 1.1 & 373 & 0.3 \\
\hline & & $21.5(14.6)$ & $20.6(13.6)$ & -0.5 & 359 & 0.6 \\
\hline Variable & & $\begin{array}{l}\text { Religious delusions } \\
(n=87) \\
n(\%)\end{array}$ & $\begin{array}{l}\text { Other delusions } \\
(n=296) \\
n(\%)\end{array}$ & $\chi^{2}$ & $d f$ & $p$ \\
\hline Reasoning biases & & & & & & \\
\hline JTC on $85: 15$ tas & & & & & & \\
\hline Yes & & $36(55)$ & $108(47)$ & 1.3 & 2 & 0.2 \\
\hline No & & $30(45)$ & $124(53)$ & & & \\
\hline JTC on $60: 40$ tas & & & & & & \\
\hline Yes & & $25(38)$ & $79(35)$ & 0.3 & 1 & 0.6 \\
\hline No & & $41(62)$ & $150(65)$ & & & \\
\hline Alternative expla & anatic & & & & & \\
\hline Yes & & $27(34)$ & $52(21.1)$ & $5.6,0.1$ & 1 & 0.02 \\
\hline No & & $52(66)$ & $195(78.9)$ & & & \\
\hline $\begin{array}{l}\text { Possibility of beir } \\
\text { mistaken }\end{array}$ & ing & & & & & \\
\hline Yes & & $41(50)$ & $123(48)$ & 3.9 & 2 & 0.1 \\
\hline No & & $41(50)$ & $133(52)$ & & & \\
\hline Variable & & $\begin{array}{l}\text { Religious delusions ( } n=87 \\
\text { Mean (SD) }\end{array}$ & $\begin{array}{l}\text { Other delusions }(n=296 \\
\text { Mean (SD) }\end{array}$ & 6) & $d f$ & $p$ \\
\hline Illness perceptions ${ }^{\mathrm{a}}$ & & & & & & \\
\hline IPQ cure/control & & $21.2(4.3)$ & $20.5(405)$ & -1.0 & 221 & 0.3 \\
\hline IPQ timeline & & $9.7(3.5)$ & $9.8(3.3)$ & 0.2 & 224 & 0.8 \\
\hline IPQ state of minc & & $3.6(1.3)$ & $3.6(1.3)$ & -0.2 & 243 & 0.9 \\
\hline IPQ personality & & $3.4(1.3)$ & $3.4(1.3)$ & -0.1 & 243 & 0.9 \\
\hline Variable & $\begin{array}{l}\text { Reliq } \\
\text { Mea } \\
(n=\end{array}$ & $\begin{array}{l}\text { gious delusions }(n=87) \\
\text { n }(\mathrm{SD}) \\
58)\end{array}$ & $\begin{array}{l}\text { Other delusions }(n=296) \\
\text { Mean }(\mathrm{SD}) \\
(n=175)\end{array}$ & $t$ & $d f$ & $p$ \\
\hline Insight scale ${ }^{a}$ & 8.3( & & $8.2(3.9)$ & -0.5 & 231 & 1.0 \\
\hline
\end{tabular}

variable. The final model (step 5) was a good fit $\left(\chi^{2}=67.6, d f=6, p<0.001\right)$, with an estimated pseudo $r^{2}$ of 0.3 . Grandiose delusions, delusions of being controlled, Internal evidence and access to an alternative explanation each independently increased the likelihood of having a religious delusion, with effect sizes ranging from over seven times as likely, to twice as likely. Each step increase in avolition/apathy scores reduced the likelihood 
Table 5 Final model of the binary logistic regression analysis illustrating the predictors of religious delusions

\begin{tabular}{lllc}
\hline Independent variable & OR & $95 \%$ CI & $p$ \\
\hline Grandiose delusions & 7.5 & $3.9-14.1$ & $<0.001$ \\
Delusions of being controlled & 3.2 & $1.5-6.6$ & 0.002 \\
Bizarre behaviour & 1.3 & $1.0-1.7$ & 0.07 \\
Avolition/apathy & 0.8 & $0.6-1.0$ & 0.02 \\
Alternative explanations & 2.2 & $1.1-4.2$ & 0.02 \\
Internal evidence & 3.4 & $1.3-8.6$ & 0.01 \\
\hline
\end{tabular}

$O R$ odds ratio, $C I$ confidence intervals

of having a religious delusion by $20 \%$. Bizarre behaviour made a small contribution, at a trend level. Results are presented in Table 5.

\section{Discussion}

We set out to examine the psychological correlates of the higher levels of persistence, distress and disability reported in the literature to be associated with religious delusions. Our aim was to understand the perceptual, emotional, cognitive, and behavioural processes underlying the treatment resistance, to better inform cognitive behavioural interventions.

In this large sample, around a fifth of delusions was religious in content. We found that religious delusions were associated with higher levels of positive symptoms, auditory and other hallucinations, thought disorder, bizarre behaviour and passivity phenomena. People with religious delusions also reported more internal evidence for their delusions (anomalous experiences or mood changes), and were very likely to have an accompanying grandiose delusion. In contrast to findings in the literature $[1,3,4$, 13-19], they had lower levels of negative symptoms, with no differences in their degree of delusional conviction or in the likelihood of them engaging in treatment. Levels of affective disturbance were similar in RD compared to other delusions, and reasoning biases were, if anything, less pronounced in the religious delusions group, as people with religious delusions were more likely to be able to identify an alternative to their delusion. The groups did not differ in their levels of insight, engagement or in their beliefs about treatment.

It is possible that by selecting participants for the current study who were already to some degree treatment resistant (history of relapse, or of symptom persistence), some of the differences found between those with religious delusions and those with other delusions in studies based on unselected samples were minimised. Nevertheless, our findings suggest that levels of positive symptoms, and specifically of grandiosity and anomalous experiences, including passivity phenomena, are elevated in people with religious delusions, even when compared to an otherwise similarly 'unwell' group. These characteristics could plausibly underlie the persistence of religious delusions and their resistance to treatment. There was no evidence that any other hypothesised maintaining factor was differentially elevated, or that beliefs about treatment were more negative in the religious delusions group. This is surprising as grandiose beliefs were prominent in the group, and are characterised by a greater likelihood of reasoning biases [47]. As with accompanying persecutory delusions in Garety and colleagues' study, it is possible that accompanying religious delusions act to moderate the cognitive and affective biases that are characteristic of grandiose delusions. The religious delusions group overall was no more likely to experience paranoid delusions than the group with no religious delusions.

Greater grandiosity may in itself be a block to treatment [1]; in that professionals may be hesitant to intervene because of the apparently protective effects of the delusion, or because of low levels of distress. Nevertheless, despite the co-occurrence with grandiosity, our findings suggest that beliefs about treatment and engagement are no different in people with religious delusions, compared to any other delusion, and, therefore, that a range of interventions should be offered. Indeed, the greater likelihood of generating an alternative to the beliefs raises the possibility that people with religious delusions may be particularly amenable to cognitive behavioural therapy. There was no evidence from our sample to suggest that this, or any other treatment offered, would be particularly unacceptable to a religious delusions group.

Considering the severity of psychotic symptomatology amongst religiously deluded patients, they may also benefit from being offered a review of their medication. Despite experiencing positive symptoms to a greater degree, medication levels, measured by CPZ equivalents, were no different in the religious delusions group compared to people with other kinds of delusions, and over $60 \%$ were on a 'low' or 'medium' dose of medication. This is a crude index, and may simply represent avoidance of over-prescribing, but as the group did not demonstrate poor insight, or negative attitudes to medication, the possibility of improving outcomes by optimising pharmacological interventions should also be considered, and may act synergistically with psychological therapy.

\section{Clinical implications}

We found that religious delusions were more likely to be accompanied by grandiose delusions, and high levels of positive symptomatology, including hallucinations, passivity phenomena, and unusual behaviour. Within a 
cognitive model of religious delusions, persistence of distress and disability and poorer outcomes may, therefore, be driven by high levels of ongoing evidence for the delusion in the form of anomalous experiences. It is possible, if the experiences have religious significance, that the person engages in particular behaviours to bring these experiences on. The high levels of bizarre behaviour found in our sample would be consistent with this suggestion. Bizarre behaviour may also act to alienate the person and reduce opportunities for social support and potential disconfirmation through social contact; or form a safety behaviour, preventing testing out of concerns [48, 49]. Odd behaviours may also act directly to confirm delusions by generating unusual or adverse reactions from others. High levels of grandiosity may limit the person's ability to reflect upon, and consider, both their actions, and their explanations of experiences. Grandiose delusions may have positive implications which mean the person is reluctant to change them.

Our findings suggest that in therapy with people with religious delusions, particular emphasis should be placed on the nature of ongoing evidence. Alternative explanations for this are likely to be available, but care may be required to ensure that valued and potentially self-esteem enhancing aspects of the belief, and those associated with positive religious coping [50,51], are not modified in an unhelpful way, and that interventions are genuinely collaborative and carefully targeted on distress and disability. Attentional processes are also likely to be an important target, aiming to reduce unhelpful tendencies to look out for, and to focus on, anomalous experiences. Some negotiation, and discussion of pros and cons, may be required around behaviours which are causing difficulty or placing the person at risk, if their negative impact is not recognised by the service user. The role of particular behaviours in triggering or maintaining anomalous experiences, or reducing the possibility of disconfirmation should be considered.

\section{Limitations}

This study adopted a cross sectional design and thus no causal relationships can be established. Cultural factors were not a focus of either main study, so despite their importance to RD, they could not be considered in this investigation. Multiple tests were carried out, and, although the sample size is large, only the global positive symptom, delusion and bizarre behaviour differences remain significant after Bonferroni correction. The findings should, therefore, be taken as pointers for future research, which should specifically target participants with RD to recruit in sufficiently large numbers.
Future research

Clarification of possible cultural variations in the psychological mechanisms underpinning religious delusions would be a useful area for future research. Researchers have proposed a distinction between African-Caribbean patients and other ethnic groups in their religious activity and belief levels [52], and the incidence of psychosis is itself influenced by racial and cultural characteristics [53]. Testable predictions arise from the tentative cognitive model of religious delusions proposed. Further research is required to clarify levels of engagement with and appraisals of anomalous experiences in people with religious delusions, and the impact of experiences and appraisals on behaviour. More work is needed to understand the difference between socially acceptable religious beliefs and religious delusions, particularly the factors determining the helpfulness or otherwise of a belief [54].

\section{Conclusions}

Approximately one-fifth of people with delusions have religious delusions. Their attitudes to and levels of engagement with treatment are similar to those of people with any kind of delusion, and therefore efforts should be made to optimise both psychological therapies and prescribing. Cognitive therapy may be an especially good 'fit', with adaptations to specifically target high levels of positive symptoms, particularly anomalous and passivity experiences, and their impact on behaviour, in the context of grandiosity. A cognitive model of religious delusions needs to incorporate an understanding of the differential impact of religious belief compared to religious delusion, and the role of anomalous experiences. Such experiences may be valued, rather than distressing, and care should be taken to understand and to preserve life-enhancing aspects of beliefs, to promote a personally meaningful recovery.

Acknowledgments The PRP group work was supported by a programme Grant (No. 062452) and a project Grant (No. 085396) from the Wellcome Trust. PG \& EK were part funded by the Department of Health via the National Institute for Health Research (NIHR) Specialist Biomedical Research Centre for Mental Health award to South London and Maudsley NHS Foundation Trust (SLaM) and the Institute of Psychiatry at King's College, London. DFr is supported by a Medical Research Council (MRC) Senior Clinical Fellowship.

We wish to thank the patients taking part in the studies and the participating teams in the four NHS Trusts-South London \& Maudsley NHS Foundation Trust, North East London NHS Foundation Trust, Camden \& Islington NHS Foundation Trust, Norfolk \& Waveney Mental Health NHS Foundation Trust.

Conflict of interest The authors declare that they have no conflict of interest. 


\section{References}

1. Appelbaum PS, Robbins PC, Roth LH (1999) Dimensional approach to delusions: comparison across types and diagnoses. Am J Psychiatry 156:1938-1943

2. Garety PA (1991) Reasoning and delusions. Br J Psychiatry 159:14-18

3. Cannon BJ, Kramer LM (2012) Delusion content across the 20th century in an American Psychiatric Hospital. Int J Soc Psychiatry $58: 323-327$

4. Siddle R, Haddock G, Tarrier N, Faragher EB (2002) Religious delusions in patients admitted to hospital with schizophrenia. Soc Psychiatry Psychiatr Epidemiol 37:130-138

5. Stompe T, Friedman A, Ortwein G, Strobl R, Chaudhry H, Najam N, Chaudhry M (1999) Comparisons of delusions among schizophrenics in Austria and in Pakistan. Psychopathology 32:225-234

6. Stompe T, Ortwein-Swoboda G, Ritter K, Schanda H (2003) Old wine in new bottles? Psychopathology 36:6-12

7. WHO (1993) The ICD-10 classification of mental and behavioural disorders, diagnostic criteria for research. World health Organisation, Geneva

8. Aukst-Margetic B, Jakovljevic M (2008) Religiosity and schizophrenia. Psychiatr Danub 20:437-438

9. Campion J, Bhugra D (1997) Experiences of religious healing in psychiatric patients in South India. Soc Psychiatry Psychiatr Epidemiol 32:215-221

10. Braam AW, Beekman ATF, Van Tilburg TG, Deeg DJH, Van Tilburg W (1997) Religious involvement and depression in older ducth citizens. Soc Psychiatry Psychiatr Epidemiol 32:284-291

11. Maselko J, Buka S (2008) Religious activity and lifetime prevalence of psychiatric disorder. Soc Psychiatry Psychiatr Epidemiol 43:18-21

12. Mohr S, Borras L, Betrisey C, Brandt P, Gillieron C, Huguelet $P$ (2010) Delusions with religious content in patients with psychosis: how they interact with spiritual coping. Psychiatry 73:158-172

13. Bhavsar V, Bhugra D (2008) Religious delusions: finding meanings in psychosis. Psychopathology 41:165-172

14. Huguelet P, Mohr S, Jung V, Gillieron C, Brandt P, Borras L (2007) Effect of religion on suicide attempts in outpatients with schizophrenia or schizo-affective disorders compared with inpatients with non-psychotic disorders. Eur Psychiatry 22:188-194

15. Mohr S, Perroud N, Gillieron C, Brandt P, Rieben I, Borras L, Huguelet P (2011) Spirituality and religiousness as predictive factors of outcome in schizophrenia and schizo-affective disorders. Psychiatry Res 186:177-182

16. Gearing RE, Alonzo D, Smolak A, McHugh K, Harmon S, Baldwin S (2011) Association of religion with delusions and hallucinations in the context of schizophrenia: implications for engagement and adherence. Schizophr Res 126:150-163

17. Huang CL-C, Shang C-Y, Shieh M-S, Lin H-N, Su JC-J (2011) The interactions between religion, religiosity, religious delusion/ hallucination, and treatment-seeking behavior among schizophrenic patients in Taiwan. Psychiatry Res 187:347-353

18. Mohr S, Huguelet P (2004) The relationship between schizophrenia and religion and its implications for care. Swiss Med Wkly 134:369-376

19. Moss Q, Fleck DE, Strakowski SM (2006) The influence of religious affiliation on time to first treatment and hospitalization. Schizophr Res 84:421-426

20. NICE (2009) Core interventions in the treatment and management of schizophrenia in primary and secondary care (update) (cg82). National Institute for Health and Clinical Excellence, London
21. Mohr S, Borras L, Rieben I, Betrisey C, Gillieron C, Brandt P-Y, Perroud N, Huguelet P (2009) Evolution of spirituality and religiousness in chronic schizophrenia or schizo-affective disorders: a 3-years follow up study. Soc Psychiatry Psychiatr Epidemiol 45:1095-1103

22. Garety PA, Bebbington P, Fowler D, Freeman D, Kuipers E (2007) Implications for neurobiological research of cognitive models of psychosis: a theoretical paper. Psychol Med 37:1377-1391

23. Garety PA, Kuipers E, Fowler D, Freeman D, Bebbington PE (2001) A cognitive model of the positive symptoms of psychosis. Psychol Med 31:189-195

24. Garety PA, Freeman D (2013) The past and future of delusions research: from the inexplicable to the treatable. Br J Psychiatry 203:327-333

25. Freeman D, Dunn G, Fowler D, Bebbington P, Kuipers E, Emsley R, Jolley S, Garety P (2013) Current paranoid thinking in patients with delusions: the presence of cognitive-affective biases. Schizophr Bull 39:1281-1287

26. Freeman D, Garety PA (2003) Connecting neurosis and psychosis: the direct influence of emotion on delusions and hallucinations. Behav Res Ther 41:923-947

27. Watson PW, Garety PA, Weinman J, Dunn G, Bebbington PE, Fowler D, Freeman D, Kuipers E (2006) Emotional dysfunction in schizophrenia spectrum psychosis: the role of illness perceptions. Psychol Med 36:761-770

28. Freeman D, Dunn G, Garety P, Weinman J, Kuipers E, Fowler D, Jolley S, Bebbington P (2012) Patients' beliefs about the causes, persistence and control of psychotic experiences predict take-up of effective cognitive behaviour therapy for psychosis. Psychol Med 10:1-9

29. Ghane S, Kolk AM, Emmelkamp PM (2010) Assessment of explanatory models of mental illness: effects of patient and interviewer characteristics. Soc Psychiatry Psychiatr Epidemiol 45:175-182

30. Garety PA, Fowler DG, Freeman D, Bebbington P, Dunn G, Kuipers E (2008) Cognitive-behavioural therapy and family intervention for relapse prevention and symptom reduction in psychosis: randomised controlled trial. $\mathrm{Br} \mathrm{J}$ Psychiatry 192:412-423

31. Wing JK, Babor T, Brugha T, Burke J, Cooper JE, Giel R, Jablenski A, Regier D, Sartorius N (1990) Scan. Schedules for clinical assessment in neuropsychiatry. Arch Gen Psychiatry 47:589-593

32. Andreasen NC (1984) Scale for the assessment of positive symptoms (saps). The University of Iowa, Iowa City

33. Andreasen NC (1984) Scale for the assessment of negative symptoms (sans). The University of Iowa, Iowa City

34. Haddock G, McCarron J, Tarrier N, Faragher EB (1999) Scales to measure dimensions of hallucinations and delusions: the psychotic symptom rating scales (psyrats). Psychol Med 29:879-889

35. Failko L, Garety PA, Kuipers E, Dunn G, Bebbington PE, Fowler DG, Freeman D (2008) A large-scale validation of the Medication Adherence Rating Scale (mars). Schizophr Res 100:53-59

36. Dunn G, Fowler D, Rollinson R, Freeman D, Kuipers E, Smith B, Steel C, Onwumere J, Jolley S, Garety P, Bebbington P (2012) Effective elements of cognitive behaviour therapy for psychosis: results of a novel type of subgroup analysis based on principal stratification. Psychol Med 42:1057-1068

37. Hall M, Meaden A, Smith J, Jones C (2001) The development and psychometric properties of an observer-rated measure of engagement with mental health services. J Ment Health 10:457-465

38. Beck AT, Steer R, Brown GK (1996) Bdi-ii manual. Psychological Corporation, San Antonio 
39. Beck AT, Epstein N, Brown G, Steer R (1988) An inventory for measuring clinical anxiety: psychometric properties. J Consult Clin Psychol 56:893-897

40. So SH, Freeman D, Dunn G, Kapur S, Kuipers E, Bebbington P, Fowler D, Garety PA (2012) Jumping to conclusions, a lack of belief flexibility and delusional conviction in psychosis: a longitudinal investigation of the structure, frequency, and relatedness of reasoning biases. J Abnorm Psychol 121:129-139

41. Wessely S, Buchanan A, Reed A, Cutting J, Everitt B, Garety PA, Taylor EJ (1993) Acting on delusions. 1: prevalence. Br J Psychiatry 163:69-76

42. Freeman D, Garety PA, Fowler D, Kuipers E, Bebbington PE, Dunn G (2004) Why do people with delusions fail to choose more realistic explanations for their experiences? An empirical investigation. J Consult Clin Psychol 72:671-680

43. Taylor PJ, Garety PA, Buchanan A, Reed A, Wessely S, Ray K, Dunn G, Grubin D (1994) Delusions and violence. In: Monahan J, Steadman HJ (eds) Violence and mental disorder: developments in risk assessments. Chicago University Press, Chicago

44. Garety PA, Freeman D, Jolley S, Dunn G, Bebbington PE, Fowler D, Dudley R (2005) Reasoning, emotions, and delusional conviction in psychosis. J Abnorm Psychol 114:373-384

45. Weinman J, Petrie KJ, Moss-Morris R, Horne R (1996) The illness perception questionnaire: a new method for assessing the cognitive representation of illness. Psychol Health 11:431-445

46. Amador XF, Strauss DH, Yale S, Gorman JM, Endicott J (1993) The assessment of insight in psychosis. Am J Psychiatry 150:873-879

47. Garety PA, Gittins M, Jolley S, Bebbington P, Dunn G, Kuipers E, Fowler D, Freeman D (2012) Differences in cognitive and emotional processes between persecutory and grandiose delusions. Schizophr Bull 12:12

48. Freeman D, Garety PA, Kuipers E, Fowler D, Bebbington PE, Dunn G (2007) Acting on persecutory delusions: the importance of safety seeking. Behav Res Ther 45:89-99

49. Jolley S, Ferner H, Bebbington P, Garety P, Dunn G, Freeman D, Fowler D, Kuipers E (2013) Delusional belief flexibility and informal caregiving relationships in psychosis: a potential cognitive route for the protective effect of social support. Epidemiol Psychiatr Sci (in press)

50. Pargament KI, Lomax JW (2013) Understanding and addressing religion among people with mental illness. World Psychiatry: Off J World Psychiatr Assoc (WPA) 12:26-32

51. Rosmarin DH, Bigda-Peyton JS, Öngur D, Pargament KI, Björgvinsson $\mathrm{T}$ (2013) Religious coping among psychotic patients: relevance to suicidality and treatment outcomes. Psychiatry Res 210:182-187

52. Littlewood R, Lipsedge M (1997) Aliens and alienists: ethnic minorities and psychiatry, 3rd edn. Brunner-Routledge, Great Britain

53. Boydell J, Bebbington $\mathrm{P}$, Bhavsar V, Kravariti E, van Os $\mathrm{J}$, Murray RM, Dutta R (2013) Unemployment, ethnicity and psychosis. Acta psychiatrica Scandinavica 127:202-207

54. Koenig HG, Zaben FA, Khalifa DA (2012) Religion, spirituality and mental health in the west and the middle east. Asian J Psychiatry $5: 180-182$

55. Cohen J (1992) A power primer. Psychol Bull 112:155-159 